\title{
La confianza pública en Hannah Arendt
}

\author{
ERIC POMMIER* \\ Pontificia Universidad Católica de Chile (Chile) \\ epommier@uc.cl
}

\begin{abstract}
Resumen
En una primera parte, quisiera plantear de forma preliminar y breve los problemas de la confianza en la acción, en el espíritu y en la institución, tal como se los pueden formular desde el pensamiento de Arendt. En una segunda parte, quisiera enfocarme sobre la tensión interna correspondiente a la acción, lo que nos conducirá, en una tercera parte, a la cuestión del espíritu como medio de superar la tensión interna a la acción, pero también como fuente de otra dificultad que tendremos que enfrentar. En una cuarta parte y a la luz de los análisis anteriores, se podrá formular de manera más precisa la cuestión de la confianza en la institución e indagaremos en las maneras de suscitarla.
\end{abstract}

Palabras clave: Acción, confianza, espíritu, juicio, institución.

\section{Public Trust in Hannah Arendt}

\begin{abstract}
In the first part, I would like to raise the issue of trust in action, in mind, and institution from Arendt's perspective. In the second part, I will focus on the internal tension of the action. In the third part, we will see that the use of the mind is the solution to this tension but that it also raises another issue. In the last part and thanks to the previous analyses, we will be able to tackle the question of trust in the institution and how to strengthen it.
\end{abstract}

Key words: Action, institution, judgement, mind, trust.

* Doctor en Filosofía por la Universidad de Paris I - Panthéon-Sorbonne. Profesor Asociado y Director de Investigación del Instituto de Filosofía de la PUC. Entre sus publicaciones destaca el libro Ontologie de la vie et éthique de la responsabilité selon Hans Jonas (2013). Algunos de sus artículos recientes son "The Problem of History in Patočka on the Basis of an Appropriation of Arendt's Anthropology" (2020), "Ethique et politique chez Hans Jonas et Hannah Arendt" (2013, y trad. portugués brasileño en 2016), "La posibilidad de la historia en la época de la responsabilidad. Vida, historia y Ética en Hans Jonas" (2017), y “Hans Jonas’s Biological Philosophy: Metaphysics or Phenomenology?” (2017).

Este artículo ha sido redactado en el marco del proyecto Fondecyt n¹181858. 
No es muy original afirmar que la situación contemporánea heredada del siglo XX se caracteriza por un cierto derrumbamiento de las ideologías encargadas de guiar el desarrollo histórico de la humanidad. Se generalizó una sospecha hacia las configuraciones de sentido tradicionales susceptibles de orientar el destino de las sociedades humanas. Las trascendencias morales, religiosas, humanistas que daban su fundamento a las instituciones políticas ya no inspiran mucha confianza. Aparecen como meras máscaras de lógicas de poder o de intereses económicos escondidas. Pero tampoco tenemos plena fe en los pensamientos "materialistas" que pretenden desvelar el motor verdadero y velado de la historia, sea en los conflictos de carácter económico o en las puras lógicas de fuerza. Estos sistemas de pensamiento parecen remitirse también a propuestas de carácter metafísico en virtud de las cuales predeterminan lo que quieren ver en la realidad socio-política (Patočka, 1999: 229).

Tampoco es muy original afirmar que este nihilismo político abre la puerta a todo tipo de populismo, de gobierno dictatorial o de neo-totalitarismo. Cuando las conciencias dejan de interesarse por la esfera pública en la cual les toca existir, abandonan la responsabilidad que ellas tienen de co-organizarla, lo que beneficia a tal o cual fuerza del momento capaz de imponer un régimen de poder favorable a sus intereses privados. La pérdida total de confianza en las trascendencias orientadoras del sentido de existencia colectiva genera un tipo de hiper confianza alucinada e ingenua en gobiernos autoritarios, confianza precaria y probablemente momentánea pero también peligrosa si tal gobierno alcanza a encarcelar las conciencias por medio del terror, de la manipulación orquestada y de la fuerza pura. El vacío del sentido no siempre significa fecundidad y promesa de renovación, pues puede anunciar a su vez, nuevas catástrofes políticas.

Es probablemente una ingenuidad creer que las trascendencias tradicionales pueden protegernos de los cataclismos políticos. Sería también un error creer que estos cataclismos sobrevienen de afuera, de manera puramente irracional como si nos fuesen exteriores e incomprensibles. La tentación del mal es interior a la humanidad y procede de su incapacidad de asumirse como humanidad. Es un riesgo interno a nuestro modo de ser.

Efectivamente, nuestra hipótesis (que se podrá desarrollar a la luz de los análisis de Hannah Arendt) es que la fuente de desconfianza hacia las instituciones requiere una reflexión sobre el modo de ser de la humanidad, es decir sobre su actuar y su pensar, en la medida en que su ser, es decir su modo de actuar y de pensar, es por sí mismo problemático y puede generar desconfianza. Dicho de otro modo: queremos mostrar que la acción hu- 
mana es, en sí misma, la fuente de una tensión interna que requiere el espíritu ${ }^{1}$ para ser superada. Pero el espíritu no se articula de manera evidente con la acción. Por ello, hay que dilucidar las condiciones de posibilidad de esta articulación: solo bajo dichas condiciones se podrá entender cómo las instituciones públicas pueden realmente suscitar una auténtica confianza.

Por ello, en una primera parte, quisiera plantear de forma preliminar y breve los problemas de la confianza en la acción, en el espíritu y en la institución, tal como se pueden formular desde el pensamiento de Arendt. En una segunda parte, quisiera enfocarme sobre la tensión interna correspondiente a la acción, lo que nos conducirá, en una tercera parte, a la cuestión del espíritu como medio de superar la tensión interna a la acción, pero también como fuente de otra dificultad que tendremos que enfrentar. En una cuarta parte y a la luz de los análisis anteriores, se podrá formular de manera más precisa la cuestión de la confianza en la institución e indagaremos en las maneras de suscitarla.

\section{PRIMERA FORMULACIÓN DE LOS PROBLEMAS DE LA ACCIÓN, DEL ESPÍ- RITU Y DE LA INSTITUCIÓN DESDE ARENDT}

Tenemos que aclarar de forma preliminar y breve lo que se debe entender por humanidad, a fin de identificar las tensiones de las cuales ella es el lugar y que pueden suscitar una desconfianza hacia si misma de forma tal que la institución amplifica esta desconfianza en lugar de crear las condiciones de una confianza pública.

En el marco del pensamiento de Arendt, no hay que entender por humanidad una Idea de la razón o un concepto producido por generalización a partir de casos empíricos. No es una abstracción, sino más bien una

1 Para Arendt, el espíritu (mind) es el lugar de tres actividades de base (el pensamiento, la voluntad y el juicio.) Se caracterizan por su autonomía: el desarrollo de estas actividades no necesita ninguna condición, es decir, son espontaneas. La única condición negativa que se puede mencionar para su buen desarrollo es la calma de las pasiones. Por lo esencial, esta actividad es invisible, en la medida en que conduce a retraerse del mundo fenomenal. Se pone a distancia de la presencia sensible del mundo gracias a un trabajo de abstracción, permitido por la memoria, la imaginación y la conceptualización. Este proceso de "desensorialización" (desense) de las representaciones hace posible una manifestación del espíritu a sí mismo, lo que Arendt llama también solitud (solitude) en la cual el sujeto se hace compañía a sí mismo, y que ella distingue de la soledad (loneliness) en la cual él está privado de esta compañía. El espíritu es necesariamente activo, lo que le opone al alma (soul) que es pasiva, informada por los sentidos. Por ende, no se puede decir que el espíritu es una propiedad del ser humano. Se manifiesta cada vez que se activa y entra necesariamente en conflicto con el sentido común que se ejerce en el seno del mundo sensible y visible (Arendt, 1978: 69-92) 
manera de ser en el mundo que se puede describir como tal y que tal, contempla las alternativas de asumirse o huir de sí. Me parece que la apuesta filosófica de Arendt es precisamente esta: describir el ser del ser humano por sí mismo y, en la medida en que este ser es el de una subjetividad que surge, se desarrolla y actúa en el seno de una pluralidad, hace falta efectuar una fenomenología de lo que, posteriormente, la autora denomina lo político. Dicho de otro modo, importa describir la esfera política por sí misma, sin entenderla a partir de categorías metafísicas previas y sin reducirla a infraestructuras económicas, psicológicas o físicas. Hay una autonomía de lo político porque existe una manera de entender al ser de la humanidad desde sí mismo, es decir desde su poder de actuar.

Para Arendt describir la condición humana requiere describir la manera con la cual cada uno puede afirmar y descubrir el sentido de su existencia individual en el seno de una colectividad que le asegura su realidad y a la cual su contribución le da consistencia. Solo una pluralidad hace posible la existencia de alguien y solo individuos singulares pueden mantener la existencia de esta pluralidad. Dicho de otro modo, existe en los individuos un deseo de sentido que se actualiza de manera conjunta con los demás. Por ende, parece que el sentido de mi existencia es co-instituido en un proceso intersubjetivo, de manera que no hay la posibilidad de ser el maestro solipsista de mi existencia ni tampoco que la colectividad humana sea capaz de existir por fuera de la participación de cada uno. Para existir auténticamente, el sujeto no tiene otra opción que la de inscribirse en la pluralidad y para mantenerse como humana, la esfera pública no puede eximirse de integrar las individualidades de todos.

(a) No obstante, tenemos fuertes razones también para dudar de la posibilidad de realizarse como ser singular en el seno de una pluralidad. En primer lugar, ¿no existe una contradicción esencial entre el deseo de ser sí mismo y la condición de posibilidad "social" de concretización de este deseo? ¿Cómo se puede afirmar su singularidad si cada uno busca igualmente la afirmación de esta singularidad? ¿Cuál puede ser el vínculo entre entidades que no poseen equivalentes? ¿Cómo el sentido individual puede salir de la insularidad de la conciencia para hacerse real sin oponerse de manera polémica a las otras conciencias? O bien: ¿Cómo la colectividad podría ser otra cosa que el lugar de la abolición de toda individualidad? $¿$ ¿No habríamos de elegir entonces, entre el caos de reivindicaciones particulares infinitas y el espectro de una sociedad homogeneizante? En este punto reside precisamente el problema filosófico de una humanidad que no puede recibir su esencia de una instancia previa a la cual ella podría abandonarse en virtud de un saber presupuesto y que aseguraría por ade- 
lantado la unidad social (Arendt, 1988: 225-226) y tampoco esta humanidad puede construir de manera artificial, arbitraria o relativista sus normas salvo a dejar "afuera" una parte de ella misma (Butler, 2015: 50). "La" humanidad no tiene elección. Por ser compuesta de sujetos singulares y por ser plural, necesita constituir su sentido en un proceso intersubjetivo de institución que puede ser también la condición de su destrucción. Cada uno depende de los demás para existir en nombre propio pero cada uno sabe también que los demás podrían comprometer esta vocación a existir de manera singular. Dicho de otro modo, ningún saber previo puede eximirnos de inscribirnos en la pluralidad para existir y para poder descubrirnos, pero no tenemos ninguna certeza de que esta apuesta sea un éxito y que tal pluralidad pueda salvarnos de la insignificancia o de la pérdida de sí mismo. Por ello, el problema de la humanidad, entendida desde su modo de ser político, conduce necesariamente al problema de la confianza pública. No puedo saber de antemano cómo debe ser organizada la esfera pública ni tampoco quién soy yo, pero no tengo otra opción más que inscribirme en ella para que pueda existir como ser singular y para co-constituir el sentido de esta comunidad.

Pero, la obligación de confiarse a ella por falta de poseer un saber previo no significa que esta confianza sea pasiva y ciega; como si hubiera que aceptar cualquier sentido de parte de los demás, lo que conduciría a la desaparición de un yo personal y de una pluralidad, pero tampoco significa que ella sea construida de manera arbitraria; como si cualquier sentido tuviera por sí mismo una legitimidad a priori para imponerse en la polis y guiar o determinar su desarrollo. En efecto, este sentido debe ser puesto a prueba para que haga posible la convivencia de todos los sujetos políticos. Entonces, esta exigencia nos obliga a postular que la confianza, que no es recibida ni construida arbitrariamente, es co-constituida en un proceso intersubjetivo que reconoce la existencia del conflicto (López Merino, 2015: 108-109). Es una confianza que se conquista y adquiere a través de un proceso de confrontación pacífico con los demás, lo que implica siempre la posibilidad de la traición. De tal manera, cada uno puede asegurarse su propia realidad y la del mundo ${ }^{2}$. Es así como la primera aporía surge: si la tesis es que la libertad de definirse a sí mismo en el marco de una organización política que determina libremente sus reglas de convivencia, es la

2 Es un proceso a través del cual se hace valer su amor propio (amor de sí mismo, es decir deseo de ser sí mismo) y su amor mundi. Como dice Arendt: "In the last analysis, the human world is always the product of man's amor mundi" (Arendt, 2005: 203). Es un proceso que toma el riesgo de enfrentarse a la posibilidad de la nada política o de la destrucción del espacio social, posibilidad o fragilidad que asegura paradójicamente la fuerza del espacio democrático, como si fuese la conciencia de la fragilidad de las relaciones humanas que podría preservar la posibilidad de su dimensión relacional. 
acción en virtud de la cual los agentes públicos pueden asegurar su ipseidad, entonces hay que reconocer que en ello, corren a su vez el riesgo de perderse a sí mismos. Es este primer aspecto del problema de la acción el que desearíamos enfrentar con la meta de replantear el de la confianza pública.

(b) No obstante, el problema no se encuentra solamente en torno al carácter inseguro de la acción pública, que hace necesario pero que también hace problemático el recurso a la confianza. Él se encuentra además en el espíritu. Si la existencia pública puede asegurar mi existencia o, al contrario, aniquilarla, si esta vida pública obedece a dinámicas de aseguramiento y de debilitamiento de la ipseidad, habrá que averiguar si disponemos de una facultad espiritual que nos pueda proteger contra el riesgo de desindividulizacion o de debilitamiento de sí mismo que surge del espectro de la homogeneización social, del espíritu de exclusión y de discriminación, de la falta de atención a la singularidad de las situaciones sociales que la comunidad tiene que organizar. Por ende, habrá que averiguar si existe esta facultad que nos vuelva capaces de evaluar de manera singular las situaciones, de manera tal que podamos codefinir un sentido público aceptable para todos y que tome en cuenta lo particular de los casos examinados. Habrá que ver si esta facultad del espíritu nos previene contra los riesgos de desaparición de sí mismo como ipseidad y de debilitamiento y destrucción del espacio público, como es el caso, por ejemplo, de la situación extrema del totalitarismo. Habrá que ver si hay medios espirituales para asegurarse del éxito de la acción en este proceso de singularización de los sujetos y de mantención de la pluralidad. Pero ¿el espíritu es capaz de moldearse a lo particular de las situaciones para entenderlas a partir de ellas mismas? ¿él es acaso capaz de orientar la acción, mientras su propia vocación es la de retirarse de la vida pública para alejarse de los prejuicios que se encuentran en ella y apuntar a lo universal? $\mathrm{Al}$ pensar la vida activa a partir de lo teórico, ¿no se pierde lo esencial de la vida activa? pero, ¿cómo se podría actuar de manera sensata en el mundo sin recurrir al espíritu que nos aleja de él y nos pone en posición de espectador?

Dicho de otro modo, otra fuente de desconfianza se puede encontrar a nivel del espíritu mismo. A modo de prueba radical: ¿puede éste, sin contradecirse, evaluar lo singular de las situaciones prácticas y ofrecer las garantías necesarias para no sucumbir en un periodo totalitario? Todo pasaría como si el espíritu pudiese ser el lugar de una posible contradicción en el que la humanidad tiene que asumirse para restaurar la confianza en sí misma. De hecho, por una parte, lo necesita para evaluar de forma correcta las situaciones, pero, por otra, él parece ser incapaz de moldearse a lo singular de la acción. 
(c) Pero incluso suponiendo que exista una dimensión del espíritu, a saber el juicio fecundado por el pensar y capaz de orientar la acción sin cancelar su carácter contingente, queda la pregunta sobre cómo estimular y fortalecer esta actividad de juicio en el espacio público. Si el pensar es la condición, problemática, del juicio y si el pensar se desarrolla afuera de la polis, en el recinto privado del espíritu que dialoga, silenciosa y solitariamente, consigo mismo: ¿cómo se debe pensar la institución para que ésta aliente el desarrollo del pensar y pueda en definitiva, acoger sus frutos?

Como se ve, el problema de la confianza política, que tiene que desembocar en una reflexión sobre las condiciones concretas de encarnación institucional del pensar en la esfera pública que será abordada en la cuarta parte de este artículo, presupone, en primer lugar, una meditación sobre el carácter aporético de la acción, que remite él mismo, en segundo lugar, al problema de la confianza en la capacidad del espíritu de orientar la acción.

Plantear el problema de la confianza en la acción y luego el problema de la confianza en el espíritu son pasos previos para entender cómo se puede articular la acción con el pensar. Así se hace posible definir las condiciones de una verdadera confianza en la institución pública, hoy en evidente crisis.

\section{LA CONFIANZA EN LA ACCIÓN}

En The Human Condition, Arendt procede, gracias a una fenomenología de las actividades, a una descripción de la categoría de la acción sin que ésta sea subordinada a la primacía de la teoría. Dicho de otro modo, para aprehender la acción desde sí misma, hay que emanciparla de la teoría y distinguirla de dos otras actividades, la de la labor y la del trabajo.

La labor es un imperativo vital ya que el ser humano tiene que vivir y, por ende, sacrificar su existencia a su mantención vital. Él es un metabolismo como cualquier animal y debe seguir viviendo como cualquier especie. A este nivel, no puede esperar más que una vida anónima o impersonal. Su nacimiento biológico le impone la labor para poder consumir y sobrevivir y esta imposición, es la de una necesidad vital que contradice su vocación a la libertad. Pero es también esta vocación que contradice su mero destino vital lo que puede darle un sentido. En efecto, el trabajo procede de la libertad del ser humano que puede traer a la existencia objetos artificiales. Él concibe, elabora planes que él ejecuta. Él es capaz de iniciativas, de representarse modelos y de cumplirlos. Es así como el ser humano puede crear artificialmente un mundo estable de objetos que no se consumen, sino que se usan. El sentido adviene al mundo o, adviene un 
mundo - y acá Arendt se inspira probablemente de la concepción heideggeriana de mundo como conjunto de utensilios (Zeuge) (Villa, 1966: 137138)_ pues mi existencia adquiere un sentido que la mera vida le negaba por consumirse incesantemente en un ciclo cerrado de elaboración y absorción. Sin embargo, si bien no habría la posibilidad de ser sí mismo sin los recursos de su mantención vital y sin la tela de fondo de un mundo estable dotado de sentido, tampoco ellos son suficientes para que haya una humanidad como tal, es decir para que pueda existir seres singulares. El mundo del trabajo expresa un poder libre de ejecución y una capacidad de representación, pero está también sometido a la lógica de la razón instrumental. Una existencia no puede afirmarse singularmente en este marco, pues ella no es otra cosa que la portadora de una competencia que cualquier otra podría encarnar. Por decirlo de otra manera, en la esfera de la labor y en el mundo del trabajo, las subjetividades todavía son invisibles o, en todo caso, no aparecen como ipseidades.

Solo la acción, junto con la palabra, da la posibilidad al ser humano de aparecer en persona pues, le da la oportunidad de decidir el sentido de su existencia, de hacerla aparecer ante sus propios ojos y a los de los demás. La acción manifiesta la contingencia que habita el ser humano, es decir hace aparecer lo que la condiciona. Actuar presupone una libertad de decidir el sentido de mi existencia (que corresponde al verdadero nacimiento existencial del ser humano) y hace visible esta libertad a través de la espontaneidad que caracteriza este actuar. La acción hace aparecer el ser del ser humano al mostrar su libertad de decidir quién es. Pero esta manifestación de sí mismo presupone un espacio de apariencia en el cual tenga sentido éste sí mismo. Este espacio no puede ser un conglomerado de sujetos anónimos incapaces de reconocer la singularidad de otra existencia y que la reducen, o quieren reducirla, a lo idéntico. Tampoco pueden ser subjetividades meramente particulares, pues no existiría un vínculo entre ellas en virtud del cual se sabe que la otra subjetividad se diferencia de la mía. Por ende, hay que reconocer que todas las subjetividades son iguales en lo tocante a su capacidad de diferenciarse. En eso consiste ser un "ser humano" y es esta capacidad de reconocer que todas las subjetividades pueden diferenciarse, tener otras perspectivas que la nuestra, lo que garantiza nuestra pertenencia a una misma humanidad ${ }^{3}$. Pero son también estas diferencias las que aseguran el carácter singular e irreductible de las subjetividades.

Aparece el aparecer, se visibiliza la visibilidad cuando hay acciones y, por lo tanto, cuando hay interacciones, lazos, relaciones humanas. No existen las ipseidades antes de esta comunidad de aparición, antes de este

3 En este aspecto, se puede igualmente referir a la "unidad de los conmovidos" evocada por Patočka (Patočka, 1990: 78-81). 
reconocimiento igualitario del derecho de aparecer por lo que somos. El sí mismo no se devela en la acción como si él existiera antes de la acción. Por eso, la acción no es desvelamiento, sino más bien eclosión por distinción con los demás (Tassin, 2019: 64). No existe, pues no aparece, el sí mismo en el seno de la esfera laboral y económica o de la comunidad cultural (Tassin, 2003: 121-132) donde prevaldría más bien la homogeneidad, sino en el mundo político. Mundo que nace al mismo tiempo que las subjetividades que se hacen aparecer al hacerlo aparecer. Advienen al mismo tiempo el espacio político, los vínculos y las identidades ${ }^{4}$ irreductibles a las comunidades prepolíticas $^{5}$

No obstante, nada asegura al actor individual que su proceso de subjetivación en el marco de un mundo plural no sea un fracaso. Si bien se puede decir que las insuficiencias de la esfera laboral y del mundo del trabajo pueden ser superados gracias al mundo político donde un sí mismo tiene la suerte de aparecer, si bien estas insuficiencias aparecen solo desde la esfera propiamente humana de la acción, hace falta reconocer que esta última esfera no escapa de contradicciones internas que pueden alienar la subjetividad de manera quizás más radical que el encierro en la labor y el trabajo. Como escribe Arendt:

$4 \quad$ Es de alguna manera lo que Manuel Tironi nos parece hacer valer cuando hace sugerencias para solucionar la crisis medioambiental y humana que ocurrió en la zona de Puchuncaví-Quintero afectada por el Complejo Industrial Ventanas. Es cierto que él enfatiza las medidas que el Estado debería tomar para resolver la crisis (cierre de las fábricas Codelco, respecto de las normas de la Organización Mundial de la Salud) y que tiende a poner énfasis en la dimensión vertical de las soluciones. Sin embargo, nos parece describir también las condiciones para que esta "zona de sacrificio" se haga espacio político en el cual los ciudadanos puedan contar su experiencia, tomar conocimiento de los mecanismos económico-científicos que están en juego, hacer valer su opinión, aparecer como tales y tratar de tomar el control de la situación. Ver Tironi (2018).

5 La fuente del sentido proviene de las acciones de una pluralidad (Tassin, 2019: 57) y es lo que puede describir una fenomenología de la acción gracias a la descosificación (Arendt, 1988: 181) y la desteorización (Arendt, 1988: 225-226; Taminiaux, 1992: 40) de la acción permitidas por una epojé. La epojé fenomenológica, que interviene acá, no reconduce el aparecer del mundo hacia la esfera de una conciencia egológica, ni siquiera a una existencia mortal sino a una pluralidad de actores. Esta epojé suspende la creencia en la existencia de subjetividades substanciales, ya definidas dentro de un grupo determinado, o que se pueden definir solitariamente. El foco de nuestra atención fenomenológica gira más bien hacia el mundo creado por las relaciones humanas descritas por sí misma según una dinámica compartida de constitución del sentido que pone en juego la definición por cada uno de su ser. Es así como una fenomenología de lo político puede hacer aparecer el carácter fenomenizador de la acción. Sobre la fenomenología de lo político de Arendt, ver también, Palomar Torralbo (2018: 191-194). 
All this is reason enough to turn away with despair from the realm of human affairs and to hold in contempt the human capacity for freedom, which, by producing the web of human relationships, seems to entangle its producer to such an extent that he appears much more the victim and the sufferer than the author and doer of what he has done. Nowhere, in other words, neither in labor, subject to the necessity of life, nor in fabrication, dependent upon given material, does man appear to be less free than in those capacities whose very essence is freedom and in that realm which owes its existence to nobody and nothing but man. (Arendt, 1988: 233-234)

Si la acción plural es la condición de nuestra humanización, es decir de nuestra singularización, podemos tener ciertas dudas y decir que podría ser también una condición de deshumanización. Desde este punto de vista, la sospecha hacia la esfera política no se debería a rasgos externos a la humanidad, a todo lo que se puede oponer a ella (su animalidad, los intereses económicos, las fuerzas de poder) y contra lo cual ella debería luchar gracias a un Ideal, cualquier sea, sino que la posibilidad de deshumanización estaría inscrita en la posibilidad misma de humanización.

En efecto, estamos obligados a reconocer que la acción plural no es para nada el lugar sereno y asegurado del cumplimiento de sí mismo. Es un mundo agonal, de conflictos y de oposiciones. Las perspectivas divergen. El sentido que inauguró una iniciativa, un proyecto práctico, se entrega al flujo de las relaciones humanas, a la contingencia, se hace historia de suerte que nada asegura que se mantenga el sentido inicial. No solo él puede modificarse, sino que puede también contradecir la intención inicial. La estructura misma de la acción abre la puerta a una desconfianza radical hacia la acción. Nací para aportar al mundo un sentido inédito, el mío, y sucede que la pérdida de control sobre él es tal, que me traiciona hasta el punto de que tengo que asumir la culpa del fracaso. Si bien la acción es experiencia encantadora del descubrimiento de quién soy yo, ella es también la experiencia de la imprevisibilidad del futuro y de la irrevocabilidad del pasado ¿Cómo no echar culpas sobre sí mismo y los demás por el fracaso de la acción? ¿Cómo no renunciar a actuar e incitar a los demás a hacer lo mismo con el fin de evitar llegar a una situación peor que en la que me encontraba inicialmente?

Angustia de la culpa pasada o de la posibilidad del fracaso, la humanidad tiene buenas razones para renunciar a la acción y desconfiar de ella, pues ella se expone al riesgo del mecanismo y de una enajenación que ella habría podido evitar al renunciar a actuar. Como escribe Arendt:

The only salvation from this kind of freedom seems to lie in non-acting, in abstention from the whole realm of human affairs as the only means to safeguard one's sovereignty and integrity as a person. (Arendt, 1988: 234) 
Sin embargo, renunciar a actuar a causa de la ambigüedad de la acción no es solamente protegerse del riesgo de la deshumanización, es también y con certeza, deshumanizarse al renunciar a lo que hace posible, a pesar de los riesgos, el aparecer de la ipseidad. Es así como la elección está entre la certeza de deshumanizarse o el riesgo de la humanización. La elección está entre la renuncia a la acción, a las iniciativas, a la co-elaboración colectiva del sentido, el aparecer de sí mismo - lo que significa el abandono al idiotismo consumerista, a la enajenación comunitarista, a las ideologías, a las configuraciones de sentido pre-determinadas- y, la aceptación del carácter contingente de la acción, que es una invitación a co-determinar su sentido. Por eso, la confianza se hace tan necesaria. Acá, la confianza no es aquella que se recibe de afuera de manera ciega. Tampoco es lo que se construye arbitrariamente sin limitaciones. Es lo que se gana al asumir el carácter plural y agonal de la acción.

¿Cuáles son entonces las condiciones de esta confianza que permite asegurar la humanidad contra el riesgo que ella es para sí misma? Como se sabe, Arendt enfatiza el papel de dos virtudes o capacidades cuyo sentido es político y no moral: la promesa y el perdón (Svampa, 2014: 83-84). El perdón hacia sí mismo y hacia los demás libera del peso del pasado y de la culpa, del encierro en sí mismo, del deseo de venganza. Reabre el futuro que había sido clausurado, hace renacer lo que había sido sepultado: un futuro que hay que cumplir. La promesa, por su lado, limita la indeterminación del futuro. Ella compromete el agente con la meta, ella da la energía para encarnar el sentido esperado sin cerrarse a la posibilidad de su redefinición. Ella relaciona el agente con su meta y lo garantiza contra la imponderabilidad cuando ésta es tóxica y disuade de actuar. A decir verdad, ella es la condición de posibilidad de los tratados, acuerdos, contratos que vitalizan las democracias (Ricoeur, 2000: 633).

Sin embargo, se podría criticar el carácter circular de esta solución. Para perdonar y prometer, para garantizarse contra el miedo y el sentimiento de culpa, para mantener viva la fe en las relaciones humanas y el sentido del futuro, para restaurarlo cuando ha sido perdido, ya debemos tener fe en las relaciones humanas y en uno mismo. Por ende, parece necesario salir de esta petición de principio gracias al uso de una facultad capaz de ayudarnos a analizar las situaciones particulares, para construir esta confianza y que no sea ciega. El perdón y la promesa no son solamente, ellas mismas, acciones, golpes de estado de la voluntad, sino que suponen también una facultad de análisis, una capacidad de distanciarse de la acción, de hacerse espectador de ella. Pero ¿dónde se pueden encontrar los criterios en virtud de los cuales analizar una situación singular sin recurrir a la teoría que la determina en función de conceptos generales y, por ende, la rigidizan? Además, ¿qué vale la fe en las relaciones humanas 
cuando el totalitarismo, por ejemplo, mostró de manera tan radical y abrupta el derrumbamiento de toda razón de espera y perdón? ¿El perdón no sería acá una falta de juicio, la pérdida del sentido común y de todo criterio, un paso suplementario - si es posible- hacia la desconfianza radicalizada hacia la humanidad?

\section{LA CONFIANZA EN EL ESPÍRITU}

Como lo sugerimos entonces, la fenomenología de la acción requiere un paso atrás, uno hacia la descripción de la actividad del espíritu ${ }^{6}$ en la medida en que éste es el que hace posible la acción al darnos los criterios de evaluación de una situación y de lo que se puede prometer o perdonar.

No obstante, si bien la intervención del espíritu es un requisito para actuar y para reabrir el campo de lo posible, éste también parece ser el lugar de una contradicción, que da espacio a una sospecha y a la posibilidad de la desconfianza ¿La actividad teórica, no es por esencia aquella que se aleja del mundo de las apariencias para pisar el suelo más sólido de las esencias y de las abstracciones? ¿cómo ella, invisible y privada - si es exacto afirmar que el pensar exige un diálogo interno del alma consiga misma- podría proporcionar cualquier ayuda en el mundo público y apareciente? Dicho de otro modo: no se entiende bien como la actividad pensante podría tener efectos pertinentes en la esfera política.

Como se ve, aún cuando el espíritu parece ser la condición necesaria de la acción, tanto para orientar su dirección como para reanimarla cuando la fe en esta ha sido destruida, tal espíritu parece ser incapaz por sí mismo de gatillar la acción. La condición de posibilidad de la acción, el recurso al espíritu, sería, por ende, también la condición de su imposibilidad. Ninguna vida pública podría generar confianza si, por principio interno, la fertilización de la acción por el espíritu no fuese posible y es esto lo que precisamente no parece ser posible. Peor aún, no es solamente que el espíritu se vuelva incapaz de fecundar la acción, sino que su pretensión de hacerlo conduce, al contrario, a destruirla. Como ya lo hemos mencionado, la fenomenología de la acción de Arendt consistió precisamente en emancipar la acción de su subordinación a la teoría. No se puede entender

6 En la introducción de The Life of Mind, Arendt señala que Kant "remained unaware of the fact that man's need to reflect encompasses nearly everything that happens to him, things he knows as well as things he can never know" (Arendt, 1978: 14). Se puede pensar, según Arendt, acerca de cualquier evento humano que adviene. En esta misma introducción, ella sugiere la existencia de una conexión entre el mal y el no-pensar, es decir, de alguna manera, una conexión entre el pensar y la posibilidad de una acción "buena". Se pregunta por lo tanto sobre lo que se "hace" cuando pensamos, aun cuando el pensar parece eximirse de cualquier acción. 
la acción desde un punto vista teórico que predetermine su curso, sino a condición de abolir su contingencia, la pluralidad de los actores y la singularidad de los sujetos.

Sin embargo, si bien la acción no es compatible con una cierta manera de representarse la actividad del espíritu, esta aserción no conduce a afirmar que todo tipo de actividad espiritual es incompatible con la acción. Al contrario, la emancipación de la acción respecto de la representación clásica de la teoría, debe conducirnos a reconsiderar la actividad espiritual de manera que ésta haga posible la acción. Si la acción necesita del espíritu para hacerse posible, entonces, la descripción de la vida espiritual no puede eximirse de una referencia a la acción. Es así como Arendt muestra, a principios de La vida del espiritu, que hay que romper con la concepción dualista que opone el mundo de las apariencias al mundo de la realidad verdadera, al cual, dicho sea de paso, tendríamos acceso gracias a la actividad teórica. En realidad, nunca salimos de las apariencias y la vida espiritual es solo un esfuerzo de comprensión que conduce a superar ciertas apariencias por otras (Arendt, 1978: 23-30). Si bien el espíritu se aleja de las apariencias de la praxis, nunca rompe con ellas y con las apariencias visibles en general. La cuestión del sentido requiere un distanciamiento de la vida activa pero no corta relación con ella. Por este motivo es que cualquier asunto práctico puede ser un pretexto para pensar. Finalmente, es una ilusión creer que la vida intelectual nos hace salir por completo de la vida política, de hecho, tal ilusión resulta el mejor medio para quedar dependiente, de manera ciega, de ella y permitir en consecuencia, sobredeterminarse ingenuamente por ella (Taminiaux, 1992: 155-175).

Por lo tanto, en lugar de ceder a la ilusión de un dualismo drástico entre la vida activa y la vida espiritual lo que conduce, o bien a despreciar la vida activa en beneficio de la vida espiritual, la cual sería entonces la única "acción” digna para la humanidad (Taminiaux, 1992: 59-60; Di Pego, 2019: 218-220), o bien a desacreditar la vida filosófica en nombre de una concepción estrechamente pragmática de la vida, es necesario reconocer el carácter conflictual y problemático de la articulación entre ambas vidas. En otros términos, es cierto que la vida espiritual reconduce a la interioridad, evoluciona en un mundo invisible a los ojos sensibles, y que requiere un distanciamiento con el mundo plural, sin embargo y puesto que el espíritu nace de él, puede tener efectos sobre él; orientar la acción, volver a la caverna que nunca ha abandonado de manera total, y así actuar de manera sensata. El espíritu ofrece recursos para hacer inteligente la acción y para darnos confianza en ella. Es cierto que se aleja de ella para entenderla, lo que le hace correr el riesgo de perder de vista su singularidad, pero es cierto también que la situación de espectador ofrece la posibilidad de actuar mejor. Como escribe Arendt: 
And although this can never directly change reality - indeed in our world there is no clearer or more radical opposition than that between thinking and doing - the principles by which we act and the criteria by which we judge and conduct our lives depend-ultimately on the life of the mind. (Arendt, 1978: 71)

En consecuencia, arribamos a lo siguiente: ¿qué tipo de actividad espiritual en específico hace inteligente la acción? O bien: ¿de qué manera el espíritu puede intervenir en lo real?

En Arendt, la articulación entre la vida práctica y la vida espiritual se encuentra a nivel de una filosofía del juicio. Si bien la vida social es el lugar de los prejuicios -estos no son simplemente algo negativo; puesto que aseguran un sentimiento de pertenencia a una determinada comunidad- la vida política, por el contrario, se caracteriza por la puesta en cuestión de tales prejuicios (Arendt, 2005: 99-101). Pero ¿qué significa esta puesta en cuestión? El análisis de una situación no requiere la aniquilación de las opiniones, sino su relativización de manera que surja una opinión bien formada sobre lo que hay que hacer. No se trata pues, de abandonar el registro de la opinión en beneficio de un hipotético saber que la aboliría; hay que reconocer que la opinión es el único medio para orientar la acción en el campo político ya que también es el campo de la contingencia. No obstante, esta opinión no debe convertirse en un prejuicio, por eso hay que ponerla en cuestión mediante a la consideración de otras opiniones que puedan ampliar la mirada sobre la situación vivida. Es a este nivel que interviene el juicio y entonces, las referencias que hace Arendt a la filosofía del juicio de Kant. Tal, no puede ser del tipo determinante, como si los criterios de la conducta en la situación singular que hay que evaluar, fuesen conocidos de antemano. El juicio es reflexivo en el sentido de que los criterios se dan solo a partir de la situación y gracias al análisis de las distintas opiniones posibles acerca de esta situación. El juicio amplía nuestra mentalidad y postula nuestra capacidad de tomar en consideración el punto de vista actual o posible de los demás. En cuanto tal, se hace imprescindible el recurso a la imaginación como capacidad de hacer presente su punto de vista. El juicio introduce la intersubjetividad o el pluralismo en la evaluación del caso (Arendt, 2005: 101-104; 1992: 68-77).

Se podría decir que es precisamente esta capacidad de juzgar la que nos da la seguridad suficiente para abandonarnos a la acción. La opinión bien formada da la valentía necesaria para exponerse en la esfera pública y declararnos por quiénes somos, aun cuando solo esta manifestación de sí mismo nos enseña justamente quiénes somos, pues la ipseidad no preexiste a la acción. Es ella la que hace posible la existencia de alguien y hace escapar del riesgo de ser nadie. 
Este valor del juicio aparece en su esplendor en situaciones históricas totalitarias, pues es en dichos contextos que la tentación a renunciar a él es fuerte. Más cuando nos enfrentamos a la tentación de renunciar a usarlo bajo el pretexto de que no se puede juzgar a los que obedecieron al poder totalitario, como denunciara Arendt, por no haberlo vivido ¿Qué manifiesta este acto de renuncia al juicio y qué se esconde detrás de él? Lo que se manifiesta no es un acto de prudencia o un gesto de humildad. Es temor y, como dice Arendt, una falta de confianza en sí mismo ("lack of selfconfidence") (Arendt, 2003: 19). Lo que se esconde es la creencia, errónea e infundada, en un mecanismo gigantesco capaz de desresponsabilizar a los seres humanos y de quitarles su libertad. Efectivamente, lo preocupante al momento de la caída totalitaria de una sociedad no es tanto las intenciones criminales de un determinado grupo, sino la renuncia generalizada de ejercer la facultad de juzgar y la disposición a aceptar las nuevas reglas de un régimen criminal, por cuyo programa no hay necesariamente adhesión (Arendt, 2003: 24). Es cierto que se puede explicar esta caída a partir de la noción de culpabilidad colectiva o de la teoría del eslabón: nadie en particular es responsable, pues la culpa es de todos en el momento en que no tienen ninguna elección sino la de obedecer. En efecto, si no hubieran obedecido, otros lo habrían hecho e, incluso, éstos habrían podido hacer más daño. Mejor entonces obedecer para que el mal sea menor. Pero el punto se vuelve el siguiente: ¿qué hace que los sujetos se conviertan en meros eslabones? ¿por qué han aceptado renunciar a su individualidad en beneficio del cumplimiento de un proyecto criminal? Si bien es exacto que el totalitarismo se caracteriza por tomar el control de la sociedad de manera tal que hay que tener presente la fuerza de la presión social al momento de comprender la obediencia de los pequeños eslabones, si bien se puede invocar el argumento del mal menor para justificar seguir asumiendo un cargo de responsabilidad en un régimen criminal, queda, sin embargo, patente que los que resistieron a la fuerza el mecanismo social, fueron los que juzgaron, es decir los que se formaron una opinión acerca de la situación que ellos vivían sin dejarse determinar por la ideología del momento y sin ceder a la inercia social. Fueron escépticos y dudaron. Es en esta actividad espiritual que encontraron los recursos para dejar de apoyar al régimen. Es gracias a esta actividad que tomaron conciencia de que obedecer era apoyar y ser co-responsable del proyecto criminal. Es gracias al juicio que pudieron saber que el poder del totalitarismo descansaba en la pasividad de los eslabones, que, al renunciar a juzgar, estos se hicieron colaboradores activos del régimen totalitario y, de reapropiarse de tal capacidad, habrían podido desmantelarlo, no en virtud de una intervención directa sino por resistencia pasiva. Como se ve, la actividad de juicio es lo 
que permite nutrir la confianza en la acción y en el ser del ser humano, haciéndose digno de sí como tal. Como dice Arendt:

If we think these matters through, we might regain some measure of self-confidence and even pride, that is, regain what former times called the dignity or the honor of man: not perhaps of mankind but of the status of being human. (Arendt, 2003: 48)

Ahora bien, si bien el juicio es la parte del espíritu que permite orientar la acción, queda por entender lo que hace posible el ejercicio del juicio, la distancia hacia la situación. Queda por entender como formar una opinión gracias a la ampliación de su mentalidad. Hace falta dilucidar lo que hace que las opiniones converjan hacia una decisión singular en lugar de yuxtaponerse entre ellas y, así, crear una confusión interior. Ante estas interrogantes, no hay otra opción que reconocer, conforme al testimonio de la experiencia, la existencia del pensamiento que hace posible el exilio interior y el diálogo del alma consigo misma. El ser humano es de tal manera que puede retirarse de la realidad para dejarla aparecer no solo según su perspectiva, sino por sí misma. Es así como él tiene la posibilidad de extraerse de los prejuicios, de crear un vacío interior a partir del cual la singularidad de la situación, pueda finalmente aparecer como tal (Dana Villa, 1999: 12). Es el pensar lo que parece hacer posible el aparecer de la situación como tal en lugar de recubrirla o privatizarla a partir de tal o cual punto de vista. Tal distancia es fecunda, pues ella hace posible el aparecer de distintas opiniones acerca de la situación, pero de manera que éstas converjan hacia una decisión forjada en lo singular de la situación. El pensar crea las condiciones para descartar las opciones que no se adecuan al contexto (Villa, 1999: 23) y obliga a inventar — de ahí el papel de la imaginación- criterios pertinentes para la situación. Como ya se lo mencionó, el pensar no se exime por completo del mundo de las apariencias, sino que hace posible una donación de sentido adecuada al contexto. Él hace posible el aparecer de lo que hay y condiciona el poder de evaluar el fenómeno para que podamos orientarlo de manera de preservar el mundo de las apariencias humanas.

En suma, no se puede preservar la pluralidad del mundo y el juego de las ipseidades entre ellas sin reconocer la existencia de una dinámica interna que provoca nuestro desdoblamiento y que nos hace finalmente, dialogar con nosotros mismos. Pensar es ser dos en uno (Arendt, 1978: 179-193), ser su propio compañero, buscar la coherencia interna, asegurarse de que no dejará de existir el amor de sí mismo a causa de decisiones no consideradas. Como lo señala en varias oportunidades Arendt, hace falta reconocer que los que pudieron resistir al régimen nazi fueron los que supieron 
que no podrían seguir viviendo para siempre con un criminal, a saber, ellos mismos en el caso posible y efectivo de colaboración con el régimen nazi. Son los que se acostumbraron a pensar. Entonces, el problema de la confianza en la acción nos reconduce hacia la confianza en la actividad judicativa del espíritu que se fundamenta, en última instancia, en la costumbre de pensar (Arendt, 2003: 189). Es cierto que los que piensan se alejan del mundo público. Sin embargo, la confianza en la acción pública y en el mundo público descansa en la certeza de que las decisiones y las acciones fueron posible en virtud de un pensar.

\section{LA INSTITUCIÓN PÚBLICA}

La dificultad se vuelve entonces más precisa y circunscrita. Si la actividad del pensar es una actividad solitaria e invisible y si la acción es necesariamente plural y visible: ¿cómo asegurarse de que la cultura del pensar influya en la vida práctica y que ésta, a su vez, favorezca la actividad del pensar? ¿No sería, acaso, la respuesta a esta interrogante el poder restituir, mantener o hacer crecer la confianza en la vida pública?

Para atender a esta preocupación, se puede enfatizar al menos tres aspectos del pensamiento de Arendt: la cuestión de la escuela ${ }^{7}$, como lugar intermedio entre la esfera privada y el mundo público, donde se puede enseñar a pensar, en particular a partir de las obras de la cultura y probablemente gracias a la filosofía; la cuestión de la prensa que contribuye a formar las opiniones a partir de hechos verificados y que hace posible la práctica del pensar acerca de la realidad (Revault d'Allonnes, 2018: 76-80;

Si nos referimos a "The Crisis in Education", Arendt muestra que la escuela tiene como función introducir el alumno al mundo. Esta meta presupone que el educador asuma el mundo, sea responsable de él, es decir -creemos- que acepta y reconoce el carácter plural, antagónico, dialógico del mundo, sus condiciones de mantención y evolución (las virtudes de perdón, promesa, prudencia, coraje etc.) En efecto, hay que conocer y asumir el mundo, por un lado, para tener presente lo que significa iniciar al alumno en el mundo y, por otro, para preservarlo contra el riesgo de innovaciones excesivas que podrían destruirlo. Pero preservar el mundo es también una condición para que el alumno pueda advenir a sí mismo como ser humano, es decir, como ser destinado a nacer y a introducir novedades en este mundo. El nuevo tiene sentido solo en relación con un mundo relativamente estable. La nueva generación es nueva en comparación con el mundo que siempre es más antiguo que ella. Desde esta perspectiva, la educación debe tener una dimensión conservadora (Arendt, 1961: 192), es decir transmitir la tradición, cuya frecuentación debe permitir el desarrollo de los talentos del alumno que lo van a distinguir (Arendt, 1961: 189). Se puede suponer que tal educación implica enseñar las obras de la cultura pues ellas invitan al desarrollo del pensar, lo cual agiliza el ejercicio de la facultad de juicio y posibilita la acción. En efecto, en The Human Condition, Arendt insiste en decir que la obra de arte es una obra (work) de carácter particular pues procede de un pensar (Arendt, 1988: 167-174). 
Heuer, 2019: 67-68) y, la cuestión de la institución democrática como tal. Quisiera desarrollar brevemente este último aspecto por su carácter de inmediato problemático En efecto, para que el pensar pueda hacer posible la acción por medio de la actividad del juicio, debe existir un espacio público donde él pueda encarnarse y que, recíprocamente, pueda propiciar este regreso a sí mismo que caracteriza al pensamiento. Sin embargo, este espacio no puede instituirse sino a través de las acciones de la pluralidad, de otro modo, este espacio sería formal, abstracto y no democrático. Es así como la actividad de pensar está supeditada a la existencia de un espacio público o de un mundo plural que la supone. La institución legítima debe fomentar el juicio y, por ende, el pensar; pero para que esta institución no sea rigidificadora, como sería el caso de un gobierno ${ }^{8}$ de sabios que mandan a los que ejecutan (Arendt, 1988: 222), ella no puede predeterminar el curso de las acciones y de las discusiones. Lo que significa que ella deriva de este curso de acciones y discusiones y, en última instancia, de un pensar ¿Cómo se puede pensar la institución para que ella fomente lo que la presupone, para que ella sea el marco de lo que trasciende cualquier marco, para que ella "organice" la posibilidad de pensar sin esterilizarse?

Arendt otorga un valor particular a la asociación voluntaria y a una de sus formas; la desobediencia civil. Esta puede ser un remedio hacia la desconfianza generada por las instituciones tradicionales, pero ¿cuándo puede suceder? Como escribe Arendt:

Civil disobedience arises when a significant number of citizens have become convinced either that the normal channels of change no longer function, and grievances will not be heard or acted upon, or that, on the contrary, the government is about to change and has embarked upon and persists in

8 En The Human Condition, Arendt procede a una crítica del concepto de gobierno. Parece que se pierde el poder cuando se lo delega, que se desvanece la legitimidad política cuando el gobierno es "representativo". En todo caso, un gobierno no puede seguir ejerciendo el poder cuando pierde la confianza pública, cuando la gente no encuentra en él el reflejo de sus preocupaciones. Respecto del gobierno del periodo de la guerra del Vietnam, Arendt escribe: "The title of our discussion, "the Impotence of Power", sounds paradoxical, but, unfortunately it's a very apt title. I don't have to go into details of our numerous problems - urban decay, rising crime rates, racial conflicts, snarled traffic and communications, including the mails, garbage collection failures (...) You may feel, as I sometimes feel, that the country seems to have fallen under a spell. Nothing works any more, except of course trips to the moon about whose necessity one can be of very different opinions. (...) It is obvious that the people in America no longer support the government, certainly not its foreign policy, specifically the Vietnam war. And it turns out that this form of government cannot truly govern without the support of the people." (Arendt, 1969: 14389 [corresponde al número de referencia de la página concernida: “The Impotence of Power." Copyright (C) 1969 by Hannah Arendt. Reprinted by permission of Georges Borchardt, Inc., on behalf of the Hannah Arendt Bluecher Literary Trust.] 
modes of action whose legality and constitutionality are open to grave doubt. (Arendt, 1970a: 74)9

En efecto, si bien se puede hablar de consentimiento tácito acerca del contrato social originario entendido como un acuerdo horizontal entre los hombres que les hace aceptar la constitución gracias a la cual pueden organizar su vida colectiva, queda la duda por saber en qué medida este consentimiento procede de una participación efectiva de todos los hombres al proceso de formación de la constitución. Es así como Arendt nos recuerda la legítima desconfianza hacia la Constitución de los Estados Unidos, hecha por todos y sin embargo excluyendo a Negros (Arendt, 1970a: 90). Es así como se puede dudar de la capacidad de la constitución de organizar una participación concreta de los ciudadanos en los procesos de decisión (tomando en cuenta el régimen de los partidos, la burocratización, etcétera.) (Arendt, 1970a: 89). Existe también el riesgo, acerca de tal o cual ley, que una mayoría oprima los derechos de una minoría, lo que significaría su exclusión del espacio político de decisión. Frente a estas posibilidades de violaciones, no basta con la indignación moral que puede ser impotente si no es capaz de movilizar la opinión pública. Es la razón por la cual se hace necesario el derecho a la desobediencia civil. No hay que temer su carácter arbitrario pues el concepto de desobediencia civil procede de la organización de una minoría en torno a decisiones comunes más que a una unión de intereses. Es un acuerdo común que no descansa en un ideal moral superior, lo que distingue esta desobediencia de la objeción de conciencia. Tampoco se puede considerar que es un acto criminal, pues éste busca la discreción, la opacidad, huyendo de la luz pública mientras que la desobediencia civil reivindica la visibilidad. Ni siquiera busca obtener un derecho de eximirse del castigo de la ley en consecuencia de su infracción. Al contrario, la quiere para poder expresar su desacuerdo fundamental. Por último, no es una amenaza para el orden democrático establecido. Su acto no es violento, ni siquiera revolucionario, sino pacífico (Tassin, 2003: 108-109).

Si estas condiciones son cumplidas, Arendt considera legítimo integrar la desobediencia colectiva a las instituciones y, así, proteger a los que desobedecen el castigo jurídico (pues el juez evalúa la conducta individual, separándola de todo lo que hace de ella un acto político (Arendt, 1970a:

9 La correspondiente cita que se encuentra en la Lecture de 1970 pronunciada por Arendt indica que no se la ha modificado de manera substancial para la publicación. Además, en tal Lecture, la primera parte de esta cita está subrayada por Arendt (1970b: 23148 [corresponde al número de referencia de la página concernida]). 
99). Es así como ella pretende compatibilizar dos exigencias que podrían parecer contradictorias entre ellas. Lo resume Verity Smith:

it may seem that the participatory elements of her idea of democracy are in tension with the constitutional, limiting, and constraining elements. But Arendt's essay "Civil Disobedience" illuminates a way in which we can see those two sides together. That is, the activity that best embodies the way we can hold together these two elements - the desire for limited constitutional government and the need for vital, active, and participatory contestation is civil disobedience. At the heart of her insight into the constitutionality of civil disobedience is Arendt's attempt to frame civil disobedience as an activity that mediates between a need for change and a need for stability. Civil disobedience is not a fully revolutionary activity -in that such disobedient dissonance accepts the general legitimacy of the system of laws. But neither is it fully counter-revolutionary - in that the civil disobedient does want to contest the way the frame of authority is interpreted and applied. In some sense, the civil disobedient engages in the essential activity of reconstitution. (Smith, 2010: 106)

La desobediencia civil no es otra cosa que una forma de asociarse voluntariamente y de tomar compromisos. Ella vitaliza la democracia, pues elabora promesas para el futuro gracias a una opinión de calidad y un acuerdo común. Desde este punto de vista, la asociación dura hasta que su pretensión sea tomada en consideración por la comunidad en su conjunto. Este derecho a la desobediencia sería, para Arendt, una manera de reanimar la democracia al promover la acción ya que ésta supone la pluralidad, la capacidad de elaborar acuerdos y de hacerse potencia política por medio de la asociación. Establecer un cuadro constitucional que alienta las asociaciones colectivas, que da un espacio a la desobediencia colectiva y a lo que se llama hoy en día "whistleblower" (Revault d'Allonnes, 2018: 105106) ${ }^{10}$ sin romper con la necesidad de seguir reglas de vida colectiva, sería para Arendt la manera de inspirar la confianza, de fomentar la acción tomando en cuenta la estructura ontológica del ser humano, cuyo ser está habitado de manera esencial por la posibilidad de la desconfianza. Es así como al restaurar espacios públicos de formación de opiniones dando lugar a decisiones públicas, se daría la oportunidad no solo de encarnar pensamientos en el espacio público sino también de fomentar el pensar.

10 Como escribe Revault d'Allonnes: "el principio generador de la sociedad democrática implica la posibilidad permanente de su sacudimiento: es en nombre de sus principios que el whistleblower informa de las disfunciones de su ejercicio y que luego entra en el campo político". Revault d'Allonnes alude también a la necesidad, en las sociedades democráticas, de proteger al "lanceur d'alerte" gracias a la ley (2018: 105). 


\section{REFERENCIAS}

Arendt, H. (1961). Between Past and Future. Eight Exercises in Political Thought. New York: The Viking Press. New York.

Arendt, H. (1969). Speeches and Writings File, 1923-1975, n.d. Essays and lectures---"The Impotence of Power", remarks and discussion, Theatre for Ideas, New York, N.Y. Hannah Arendt Papers from Library of Congress, Washington, D.C. http://memory.loc.gov/cgi-bin/ampage?colIId $=$ mharendt\&fileName $=05 / 051490 / 051490$ page.db\&recNum $=0 \&$ itemLink $=/$ ammem $/$ arendthtml $/$ mharendtFolderP05.html\&linkText $=7$

Arendt, H. (1970a). Crises of the Republic. New York: A Harvest Book, Harcourt Brace \& Company.

Arendt, H. (1970b). Speeches and Writings File, 1923-1975, n.d. Essays and lectures---"Civil Disobedience"---Lecture---1970, Hannah Arendt Papers from Library of Congress, Washington, D.C. http://memory.loc.gov/cgibin $/$ ampage?collid $=$ mharendt $\&$ file-

Name $=05 / 051110 / 051110$ page.db\&recNum $=0 \&$ itemLink $=/ \mathrm{ammem} / \mathrm{ar}-$ endthtml/mharendtFolderP05.html\&linkText=7

Arendt, H. (1978). The Life of the Mind. New York: A Harvest Book, Harcourt Brace \& Company.

Arendt, H. (1988). The Human Condition. Chicago \& London: The University of Chicago Press.

Arendt, H. (1992). Lectures on Kant's Political Philosophy. Chicago: The University of Chicago Press.

Arendt, H. (2003). Responsibility and Judgement. New York: Schocken Books.

Arendt, H. (2005). The Promise of Politics. New York: Schocken Books.

Benhabib, S. (2019). The Reluctant Modernism of Hannah Arendt. Lanham: Rowman $\&$ Littlefield publishers, inc.

Butler, J. (2015). Notes toward a performative theory of Assembly. Cambridge, Massachusetts; London, England: Harvard University Press.

Di Pego, A. (2019). En torno al pensamiento: la disputa de Hannah Arendt con Martin Heidegger. Tópicos, Revista de Filosofía, (56), 197-235.

Heuer, W. (2019). Las tentaciones de la mentira. Universitas Philosophica, 36(72), 53-70.

López Merino, M.J. (2015). Quién es quién en el espacio público: política e identidad en H. Arendt. Revista de la Academia, 20, 103-120.

Palomar Torralbo, A. (2018). Apartar la mirada del origen: la crítica a la fenomenología política de Hannah Arendt desde el pensamiento impolítico de Roberto Esposito. Isegoría. Revista de Filosofía Moral y Política, (58), 185-204.

Patočka, J. (1999). Essais hérétiques sur la philosophie de l'histoire. Lagrasse: Verdier.

Revault d'Allonnes, M. (2018). La faiblesse du vrai. Ce que la post-vérité fait à notre monde commun. Paris: Seuil.

Ricoeur, P. (2000). La mémoire, l'histoire, l'oubli. Paris: Seuil.

Smith, V. (2010). Dissent in Dark Times. Hannah Arendt on Civil Disobedience and Constitutional Patriotism. In R. Berkowitz, J. Katz, \& T. Keenan (Eds). 
Thinking in Dark Times. Hannah Arendt on Ethics and Politics. New York: Fordham University Press.

Svampa, L. (2014). Notas sobre la promesa en el pensamiento de Friedrich Nietzsche y Hannah Arendt. Tópicos, Revista de Filosofía, (46), 75-93.

Taminiaux, J. (1992). La fille de Thrace et le penseur professionnel. Arendt et Heidegger. Paris: Payot.

Tassin, E. (2003). Un monde commun. Pour une cosmo-politique des conflits. Paris: Seuil.

Tassin, E. (2019). Une phénoménologie de l'action plurielle La phénoménologie à l'épreuve du politique, Études phénoménologiques, 3. Leuven: Peeters.

Tironi, M. (2018). Vidas tóxicas. El Mostrador. 31 de agosto. https://m.elmostrador.cl/noticias/opinion/columnas/2018/08/31/vidas-toxicas/

Villa, D. R. (1966). Arendt and Heidegger. The Fate of the Political. New Jersey: Princeton University Press.

Villa, D. R. (1999). Thinking and Judging. In J.J. Hermsen, \& D.R. Villa (Eds). The judge and the Spectator. Hannah Arendt's Political Philosophy. Leuven: Peeters. 